\title{
Congresos, Cursos y Reuniones
}

+ 13-16 junio 2009. Madrid

SEMANA DE LAS ENFERMEDADES DIGESTIVAS

Sede: Centro de Convenciones Norte. IFEMA

LXVIII Congreso Nacional de la SEPD

XVI Curso de postgrado AGA-SEPD

XIII Curso de postgrado SEED-ASGE-FEED

XI Curso de Postgrado de la AED

Secretaría Técnica:

Grupo Arán de Comunicación

Castelló, $128-1^{\circ}$

28006 Madrid

Tel. 917820033 - Fax: 915615787

e-mail:congreso7@grupoaran.com

http://www.grupoaran.com

Secretaría científica:

Sociedad Española de Patología Digestiva

Francisco Silvela, $69-2^{\circ} \mathrm{C}$

28028 Madrid

Tel. 914021353 - Fax: 914021791

http://www.sepd.es

\section{+ 22-24 octubre 2009. Granada}

XL CONGRESO DE LA SOCIEDAD ANDALUZA DE PATOLOGÍA DIGESTIVA

Sede: Hotel Abades Nevada Palace.

C/ De La Sultana, 3 - 18008 Granada

Organizador: Unidad de Aparato Digestivo.

Hospital Universitario "San Cecilio".

Avenida Doctor Olóriz, 16 - 18012 Granada

http://www.juntadeandalucia.es/servicioandaluzdesalud/hsc/

Secretaría Técnica:

Congresos XXI

Gran Vía, 81 - $5^{\circ}$ Dpto. 10 - 48011 Bilbao

Tel.: 944278855 - Fax: 944278808

e-mail: info@congresosxxi.com

+ 23-24 octubre 2009. Alcázar de San Juan (C. Real) XXXII REUNIÓN DE LA ASOCIACIÓN CASTELLANA DE APARATO DIGESTIVO

Secretaría Científica:

Sección de Aparato Digestivo

Hospital La Mancha Centro
Av. Constitución, 3

13600 Alcázar de San Juan (Ciudad Real)

Tel. 926580735

Secretaría Técnica:

Viajes Iberia, S.A.

C/ Cervantes, 8

16004 Cuenca

Tel. 969232729 - Fax: 969232726

e-mail:acad2009@congresoscuenca.com

+ 21-25 noviembre 2009. Londres GASTRO 2009 (UEGW/WCOG)

Secretariat:

Hollandstrasse 14/Mezzanine

A-1020 Vienna/Austria

Phone:+43 - (01) 2123691 - Fax: +43 - (01) 212 3691-29

Sede:

ExCeL London One Western Gateway Royal Victoria Dock London E16 1XL

Es la primera vez que se realiza en una reunión científica simultáneamente la UEGW y el Congreso Mundial de Gastroenterología http://www.gastro2009.org/index.php

e-mail: secretariat@gastro2009.org

+ 27-29 noviembre 2009. Barcelona

XXXI JORNADA NACIONAL DE LA SOCIEDAD ESPAÑO-

LA DE ENDOSCOPIA DIGESTIVA

Sede: CCIB (Centro Convenciones Internacionales Barcelona)

Presidentes Comité Organizador:

Dr. José Ramón Armengol-Miró, Dr. Jaume Boix Valverde y Dr. Juan María Pou Fernández

Secretaría científica:

Hospital Universitari de Badalona Germans Trias y Pujol

Crta.del Canyet $\mathrm{s} / \mathrm{n}$

08916 Badalona. Barcelona

Tel. 934978866

E-mail: jboix.germanstrias@gencat.cat

Secretaría técnica:

Editores Médicos S.A., EDIMSA

c/ Alsasua, 16

28023 Madrid

Tel. 913768140 - Fax: 913739907

edimsa@edimsa.es 